\title{
A review of TLD's zero-count based on temperature and radiation history of them
}

\author{
Nina TUNÇEL ${ }^{1,2}{ }^{*}$, İsmail KARAKUŞ ${ }^{l}$, Ertuğrul DÜNDAR ${ }^{l}$, Özlem TOYKAN ÇIFLLIKÇİ \\ ${ }^{1}$ Akdeniz University, School of Medicine, Radiation Oncology Department, Antalya/ Turkey \\ ${ }^{2}$ Akdeniz University, Science Faculty, Physics Department, Antalya/ Turkey
}

\begin{abstract}
In order to review the background value of a group of TLD-100, the zero-count values were collected from the first reading and after seven years after using them in experimental irradiations. The zero-counts for these two conditions were collected, as well as the dependence of temperature and radiation history of this group was evaluated. This study recommended that after frequently irradiation of TLDs the rearrangement for obtaining zero-count value must be performed before using TLDs in a new irradiation examination. Regarding on memory of radiation and thermal history, the sensitivity can change after receiving a large dose of radiation and undergoing readout. Additional annealing would be necessary to restore the original sensitivity. TLDs must be used under reproducible conditions to obtain consistent results.
\end{abstract}

\section{Introduction}

Thermoluminescence (TL) or thermally stimulated luminescence (TSL) is the light emission after removal of the source of exciting energy light, x-rays, or other radiation; the free electrons may be trapped at an energy level higher than their ground state by application of thermal energy [1,2]. The transition of electrons directly from a metastable state to ground state is forbidden. The metastable state represents a shallow electron trap and electrons returning from it to the excited state require energy. This energy can be supplied in the form of heat (thermal stimulation). The probability per unit time that a trapped electron will escape from a metastable state to an excited state is governed by the Boltzmann equation [3,4]. First, the intensity of thermoluminescent emission does not remain constant at constant temperature, but decreases with time and eventually ceases altogether. Second, the spectrum of the thermoluminescence is highly dependent on the composition of the material and is only slightly affected by the temperature of heating [5]. In the usual thermoluminescence experiments, the system is treated at a temperature at which the phosphorescence intensity is low, and later heated through a temperature range where the phosphorrescence intensity is bright, until a temperature level at which all the charges have been thermally excited out of their metastable levels and the luminescence completely disappear [6,7]. The thermoluminescence emission mainly is used in solid state dosimetry for measurement of ionizing radiation dose. In order to stabilize the trap structure, annealing processes based on temperature and time pattern are used to produce the lowest intrinsic background and to obtain the highest efficiency. Before using a new thermoluminescent (TL) crystal material for dosimetric proposes, it is needed to perform a thermal treatment process, this thermal treatment consist in many steps as initialization treatment, annealing treatment and post-irradiation treatment $[5,8-10]$. This thermal treatment is used for new thermoluminescent materials which have not been used. The aim of this thermal treatment is to stabilize the trap levels, so that during subsequent uses the intrinsic background and the sensitivity are both reproducible. The time and temperature of the initialization annealing are, in general, the same as those of the standard annealing. Standard annealing process is used to erase any previous residual irradiation effect which is supposed to remain stored in the crystal after the readout. It is carried out before using the TLDs in new measurements. The aim of this thermal treatment is to bring back the trapsrecombination centres structure to the former one obtained after this procedure. At the end of annealing procedure, the dosimeters are read to check the background signal or zero-count. The background depends on the high voltage that applied to the photomultiplier tube, on the temperature stability of TL reader and also reuse of TL material [1]. The background values determined for each dosimeter have to be collected, which is memorized in a file, so that they can be used for the successive tests $[3,11,12]$. In many cases an average background value is considered for the whole batch and then subtracted from each individual reading of the irradiated TLDs. This procedure is valid when the background is very low and constant for the whole batch. Post-irradiation

Corresponding author: ninatuncel@akdeniz.edu.tr 
treatment is used to erase the low temperature peaks, if they are found in the glow curve structure. Such low temperature peaks are normally subjected to a quick thermal fading and many times this value is not been included in the readout to avoid any errors in the dose determination $[9,13]$.

\section{Aim}

In order to review the background value of a group of TLD-100, the zero-count values were collected from the first reading and after seven years after using them in experimental irradiations. Based on group average and standard deviation of reading counts, the zerocounts for these two conditions were calculated as well as the dependence of temperature and radiation history of this group was evaluated.

\section{Materials and Method}

A hundred TLD-100 type were selected for first applications on 2010. Lithium fluoride doped with magnesium and titanium ( $\mathrm{LiF}: \mathrm{Mg}, \mathrm{Ti}$ ) is manufactured in the form of solid pellets of $4.5 \mathrm{~mm}$ diameter, $0.9 \mathrm{~mm}$ thickness under the code name MTS (MTS-N Poland) was annealed in a heat- time programmed oven. The TLDs were annealed at $400^{\circ} \mathrm{C}$ for $1 \mathrm{~h}$ and $100^{\circ} \mathrm{C}$ for $1 \mathrm{~h}$ using a PTW high temperature oven (PTW Freiburg $\mathrm{GmbH}$ ) for initial temperature treatment. And before reading TLDs the pre-read heating processed was performed $\left(100^{\circ} \mathrm{C}\right.$ for $\left.1 \mathrm{~h}\right)$. The luminescent signals for zero-counts were counted by an automated TLD reader RADOS RE-2000RT (RadRro Int. GmbH Germany). The reading arrangement was based on 4 sub-groups. So, the zero-count value was obtained for these subgroups. After seven years irradiation experiments the amount of TLDs was 80 so the collection of zero-count values was done for these remnants. This process was repeated three times for obtaining consistency of zerocount values. The average, standard deviation (SD) and percent error of these values were calculated.

\section{Results}

The zero-count values for 4 sub group of TLDs on 2010 were shown in Table 1. The general use of TLDs for routine dosimetry purposes was quite straightforward. Simplification and convenience was often implemented that make operations easier for the users but may not be consistent with the optimization of TLD performance and stability because of the more complex materials in terms of its characteristics especially with regard to the electron-hole trapping centres. The various trapping centres are defined by different energy depths (or temperature), but they are not all thermally independent, so that there may be transitions among traps such that one energy trap may deplete while another is enhanced, depending on thermal effects. One of the most important influencing
Table 1. The Zero-Count values at initial use on 2010.

\begin{tabular}{|c|c|c|c|c|}
\hline \multirow{2}{*}{$\begin{array}{c}\text { Sub } \\
\text { Group }\end{array}$} & \multicolumn{4}{|c|}{ The Zero-Count on 2010 } \\
\cline { 2 - 5 } & $\mathbf{1 .}$ & $\mathbf{2 .}$ & $\mathbf{3 .}$ & $\mathbf{4 .}$ \\
\hline Average & 3539 & 3339 & 3389 & 4165 \\
\hline SD & 541 & 113 & 143 & 348 \\
\hline \% error & 15 & 3 & 4 & 8 \\
\hline
\end{tabular}

Table 2. The Zero-Count values after seven years on 2017.

\begin{tabular}{|c|c|c|c|c|}
\hline \multirow{2}{*}{$\begin{array}{l}\text { Sub } \\
\text { Group }\end{array}$} & \multicolumn{4}{|c|}{ First reading/the Zero-Count on 2017 } \\
\cline { 2 - 5 } & $\mathbf{1 .}$ & $\mathbf{2 .}$ & $\mathbf{3 .}$ & $\mathbf{4 .}$ \\
\hline Average & 9019 & 9069 & 9519 & 12362 \\
\hline SD & 1533 & 2348 & 1883 & 3022 \\
\hline \% error & 17 & 26 & 20 & 24 \\
\hline \multirow{2}{*}{$\begin{array}{l}\text { Sub } \\
\text { Group }\end{array}$} & Second reading/the Zero-Count on 2017 \\
\cline { 2 - 5 } & $\mathbf{1 .}$ & $\mathbf{2 .}$ & $\mathbf{3 .}$ & $\mathbf{4 .}$ \\
\hline Average & 6280 & 6494 & 6588 & 6805 \\
\hline SD & 1783 & 1429 & 1919 & 1635 \\
\hline \% error & 28 & 22 & 29 & 24 \\
\hline \multirow{2}{*}{$\begin{array}{c}\text { Sub } \\
\text { Group }\end{array}$} & Third Reading/the Zero-Count on 2017 \\
\cline { 2 - 5 } & $\mathbf{1 .}$ & $\mathbf{2 .}$ & $\mathbf{3 .}$ & $\mathbf{4 .}$ \\
\hline Average & 3338 & 3477 & 3673 & 4075 \\
\hline SD & 1169 & 973 & 1300 & 1233 \\
\hline \% error & 35 & 28 & 35 & 30 \\
\hline
\end{tabular}

factors are the annealing history to which the dosimeters have been subject. The recommended full anneal process from the high temperature $\left(400^{\circ} \mathrm{C}\right)$ and cool-down period to the lower temperature anneal for LiF:Mg,Ti TLD-100 type result in sever change on TLD sensitivity. In this study, it is important to be consistent and use the same method every time that the TLDs were annealed. So, the reproducibility regarding annealing process was constant. Even with care in the annealing process, these TLDs were used in radiotherapy experiments many times that irradiated on low and high energy photons along seven years. After this period, for quite objective analyzing on TLD sensitivity the zero-count values were collected three times consecutively Table 2 .

\section{Conclusions}

This study recommended that after frequently irradiation of TLDs the rearrangement for obtaining zero-count value must be performed before using TLDs in a new irradiation examination. Regarding on memory of radiation and thermal history, the sensitivity can change after receiving a large dose of radiation and undergoing readout. Additional annealing would be necessary to restore the original sensitivity. TLDs must be used under reproducible conditions to obtain consistent results.

\section{References}

1. S.W.S. McKeever, Thermoluminescence of Solids. Cambridge University Press, Cambridge, United Kingdom, 1985.

2. G.F. Knoll, Thermoluminescent Dosimeters. Section G, Chapter 19, in Radiation Detection and Measurement, Third Edition. John Wiley \& Sons, New York, 1999. 
3. Y.S. Horowitz, Thermoluminescence and Thermoluminescent Dosimetry. (ed). CRC Press, Boca Raton, Florida. 1984.

4. F.H. Attix, Introduction to Radiological Physics and Radiation Dosimetry. Wiley-VCH Verlag GmbH \& Company, 2004.

5. S.W.S. McKeever, M. Moscovitch, and P.D. Townsend, Thermoluminescence dosimetry materials: properties and uses. Nuclear Technology Publishing, Kent, England, 1995

6. R. Chen, and S.W.S. McKeever, Theory of Thermoluminescence and Related Phenomena. World Scientific Publications Singapore, 1997.

7. Y. S. Horowitz, Phys. Med.Biol. 26(5), $765-824$ (1981).

8. J.R. Cameron et al., Thermoluminescent Dosimetry. University of Wisconsin Press, Madison, Wisconsin, 1968.

9. C. Furetta and P.S. Weng. Operational Thermoluminescence Dosimetry. (World Scientific Publishing, Hackensack, New Jersey, 1998).

10. P.W. Frame, Health Phys. 87(2), 111-135 (2004).

11. C.A. Carlsson, Phys. Med. Biol. 14(1), 107-118 (1969).

12. B.K.A. Martensson, Phys. Med. Biol. 14(1), 119-130 (1969).

13. A.P. Turner and D.W. Anderson, Phys. Med. Biol. 18(1), 46-52 (1973). 